\title{
Casapoli, residencias en Coliumo, Tomé, Chile
}

\author{
Leslie Fernández e Oscar Concha*
}

http://dx.doi.org/10.22409/poiesis.1829.073081

\begin{abstract}
RESUMEN: Con el deseo de generar un espacio de conexión de nuestra ciudad y región con circuitos artísticos nacionales e internacionales, en el 2005, los artistas Rosmarie Prim y Eduardo Meissner junto a los arquitectos Sofía von Ellrichshausen y Mauricio Pezo planificaron y construyeron en Coliumo, Tomé, Casapoli. La edificación fue pensada como un espacio para dar cabida a residencias, encuentros, producción, creación, pensamiento y reflexión en torno a las artes visuales y la arquitectura, con la libertad operativa que permite ser una institución independiente. Desde su construcción ya hace doce años Casapoli ha funcionado activamente para alojar diferentes actividades, en un comienzo gestionadas por los propietarios y autores del proyecto, más adelante con la participación de curadores independientes.

PALABRAS CLAVE: Casapoli, residencias en Coliumo, artes visuales y arquitectura ABSTARCT: With the desire to create a connection space of our city and region with national and international artistic circuits, in 2005, the artists Rosmarie Prim and Eduardo Meissner together with the architects Sofía von Ellrichshausen and Mauricio Pezo planned and built in Coliumo, Tomé, Casapoli. The building was conceived as a space to accommodate residences, meetings, production, creation,
\end{abstract}

\footnotetext{
* Oscar Concha Lagos es artista visual y gestor cultural independiente. Desde los 90 a desarrollado una sistemática producción en el campo de las artes visuales de la región del Biobío, generando y participando de iniciativas autogestionadas en los cuales se potencia la colaboración y el establecimiento de redes para el arte contemporáneo local. Actualmente codirige junto a Leslie Fernández el proyecto CASAPOLI RESIDENCIAS. Ha participado en Chile y el extranjero en exhibiciones, charlas, encuentros y residencias. Vive y trabaja desde Concepción, Chile.

Leslie Fernández Barrera vive y trabaja en Concepción, Chile. Artista Visual, Licenciada en Arte Universidad de Concepción (1993) y Magíster de la Academia San Carlos, UNAM, México (2003). Actualmente es docente del Departamento de Artes Plásticas de la Universidad de Concepción y colaboradora del programa Magíster en Arte y Patrimonio de la misma universidad. Forma parte del equipo editorial de la revista Alzaprima y es codirectora de Casapoli Residencias. Desarrolla junto a un equipo, una investigación sobre Artes visuales en Concepción durante la Dictadura.
} 
thought and reflection around the visual arts and architecture, with the operational freedom that allows being an independent institution. Since its construction twelve years ago, Casapoli has actively operated to host different activities, initially managed by the owners and authors of the project, later with the participation of independent curators.

KEYWORDS: Casapoli, residences in Coliumo, visual arts and architecture

Con el deseo de generar un espacio de conexión de nuestra ciudad y región con circuitos artísticos nacionales e internacionales, en el 2005, los artistas Rosmarie Prim y Eduardo Meissner junto a los arquitectos Sofía von Ellrichshausen y Mauricio Pezo planificaron y construyeron en Coliumo, Tomé, Casapoli. La edificación fue pensada como un espacio para dar cabida a residencias, encuentros, producción, creación, pensamiento y reflexión en torno a las artes visuales y la arquitectura, con la libertad operativa que permite ser una institución independiente. Desde su construcción ya hace doce años Casapoli ha funcionado activamente para alojar diferentes actividades, en un comienzo gestionadas por los propietarios y autores del proyecto, más adelante con la participación de curadores independientes.

Pese a aparentemente ser un cubo de geometría perfecta, Casapoli fue concebida como un cuerpo orgánico, en primer lugar por lo artesanal de su construcción realizada por medio de concreto fabricado en una pequeña betonera, trasladado apenas con cuatro carretillas. Las huellas de los moldajes del entablado sin tratamiento han quedado a la vista tanto en el interior como en el exterior haciendo visible la imperfección de un molde, la ausencia del ángulo recto, tal como señalan sus autores en la publicación 89, 911. La casa se instala como un volumen macizo, sin terminaciones depuradas que con los años han ido absorbiendo las condiciones de un clima costero extremo: humedad, lluvia, neblina y sol han ido moldeando su fachada. Al habitar Casapoli, es posible percibir detalles como la textura irregular dada por el uso de madera reciclada que la conecta con la textura presente en las rocas cercanas y también con las fisuras de los acantilados. Los cambios de luz son percibidos a través de sus numerosos ventanales, por donde también ingresa una naturaleza desbordante, caracterizada por una diversidad de líneas orgánicas, fauna local y pasajera, junto a una vegetación frondosa, 
que nos recuerdan a cada momento la geografía en donde se encuentra inmersa. Desde la amplitud de visión que permite Casapoli es posible observar un paisaje en donde las aves hacen una constante demostración de su expertiz de vuelo y donde algueras, pescadores y mariscadores desafían al mar para extraer sus productos. Sólo un poco más distante, hacia el sur, es posible percibir la referencia urbana, porteña e industrial de Talcahuano y a su lado la Isla Quiriquina, un recinto militar que guarda sucesos oscuros ocurridos durante la dictadura. Sobre el mar, en constante tránsito es posible observar barcos que con distintas funciones se desplazan hacia y desde los puertos cercanos, dejando una línea de recorrido que se proyecta infinitamente sobre El Pacífico.

Desde el 2010, más específicamente posterior al terremoto y tsunami que afectaron fuertemente a esta zona, comenzamos a trabajar como directores de residencias, en primera instancia en conjunto con la agrupación Mesa8, motivados por la contingencia que significó este desastre natural. En ese complejo momento desarrollamos diferentes actividades en la Escuela Vegas de Coliumo G-449, teniendo a Casapoli como refugio, lo que nos permitió ir estableciendo gradualmente vínculos con esa comunidad educativa, especialmente con uno de sus profesores ${ }^{2}$. Las constantes visitas a la localidad significaron más adelante la generación de vínculos con los habitantes del albergue Municipal, situado transitoriamente en la parte en los altos de Coliumo, del cual hoy solo quedan pequeños vestigios.

Entre los años 2010 y 2017 han tenido lugar en Casapoli diversas actividades entre las que mencionamos: residencias de artistas y colectivos, claustros, charlas, encuentros, visitas de artistas como de personas vinculadas a las artes visuales y la cultura. Todas estas prácticas han ido contribuyendo a que Casapoli haya ido tomando un lugar en la circulación de arte contemporáneo en la región del Bíobío. Su emplazamiento nos permite hablar de nuestra geografía y territorio, de una diversidad del paisaje pero también abordar las problemáticas socioculturales que allí suceden.

En el 2011, en una residencia coordinada en conjunto con el colectivo Mesa8, recibimos a dos artistas extranjeros que fueron muy significativos en la búsqueda de conectarse con el lugar. Ambos abordaron el territorio a partir de situaciones que consideraron les permitirían desarrollar un proyecto en un mes, tiempo de extensión de la residencia, ellos fueron Christians Luna de Perú y Leonardo Herrera de Colombia ${ }^{3}$. Luna apeló en su trabajo a la condición de 

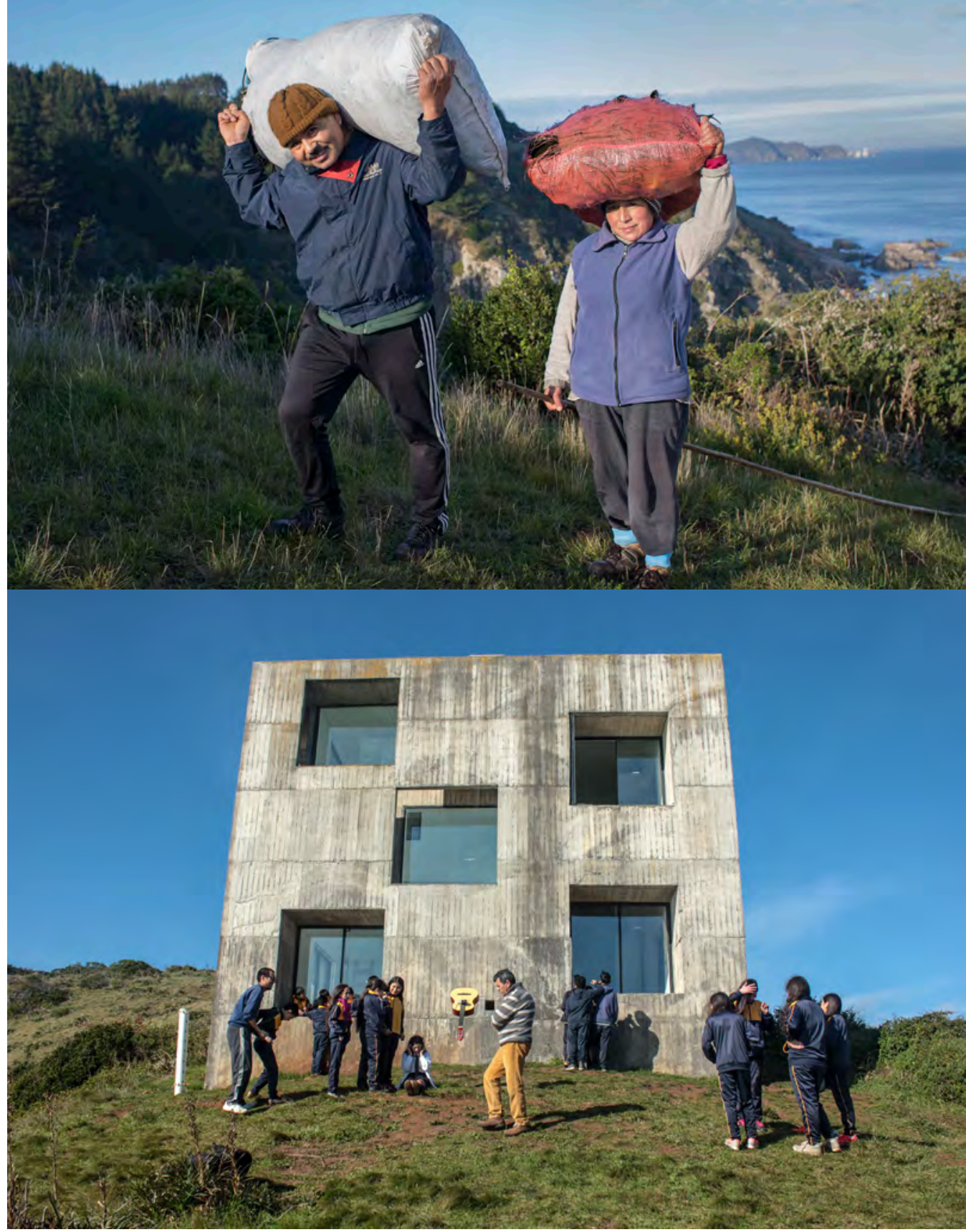

Xavier Tavera/Residencia Julio 2017, registro Xavier Tavera

Sebastian Jatz/Residencia Programa Traslado, Julio 2016, registro Sebastián Jatz 
Coliumo como una caleta de pescadores, generando a modo de juego El primer Campeonato de botecitos, pero terrestre, con la participación central de niños de la Escuela de Las Vegas y del Campamento municipal, construido en el contexto del posterremoto. Por otro lado, Leonardo Herrera tomó como punto de referencia la Industria Textil de Tomé, realizando un Encuentro de la memoria viva en donde se convocó abiertamente a tomecinos vinculados de alguna manera con esa industria, para compartir documentos, objetos y relatos que pudieran referirse al pasado reciente de Tomé, que la instaló nivel nacional como un importante centro de producción. Ambas residencias se centraron en lo colectivo, en donde el artista operó como un articulador y generador de un espacio de diálogo 4 .

En cuanto a los ciclos específicos en 2014 se desarrolló Contexto y territorio, con residencias convocadas bajo lineamientos temáticos relativos a la naturaleza y al contexto socio-cultural en donde conviven educación, oficios, alimentación, arquitectura, habitabilidad, etc. Fue un ciclo realizado con artistas invitados y seleccionados, cuyos trabajos buscaron generar una interacción con la comunidad de Coliumo, Tomé y Concepción. Otra de las motivaciones del ciclo fue potenciar vínculos con artistas locales, con el Departamento de Arte de la Universidad de Concepción y con espacios independientes de Concepción y Tomé.

Participaron del ciclo de residencias 2014 artistas invitados en consideración de la línea de trabajo que han desarrollado anteriormente más cuatro artistas jóvenes quienes presentaron propuestas específicas, las cuales fueron seleccionadas a partir de una convocatoria regional ${ }^{5}$. Del ciclo 2014 destacamos la residencia de Juan Castillo, quien desarrolló una intervención en el paisaje como continuidad del proyecto Ritos de Paso realizado anteriormente en el desierto de Atacama y Chiloé. Castillo realizó además, junto a la artista Natascha de Cortillas, una acción culinaria colaborativa que reunió en torno a una mesa a artistas de Concepción y de Tomé. También destacamos la participación Rainer Krause, artista sonoro, quien continuó desarrollando su recopilación de sonidos costeros además del proyecto $N$ consistente en la captura de voces, para lo cual requirió la colaboración de habitantes de Coliumo, Tomé y Concepción. Durante el mes de noviembre de ese mismo año Adolfo Torres, quien desde el 2000 trabaja en el proyecto La Olla Común que entrelaza el arte y la cocina popular, realizó en la caleta del Morro un Causeo comunitario público, organizado en conjunto con La cocinería El Loro del cual participaron habitantes y visitantes casuales de Coliumo. 
Durante el 2016 se realizó el ciclo Territorio compartido6, a través del cual se buscaba generar un trabajo interdisciplinar por medio de la generación de duplas constituidas por investigadores del área de las ciencias y las artes visuales. Profesionales de geología, oceanografía, antropología, biología marina e historia del arte convivieron con artistas visuales. En total fueron cinco residencias en donde no existía la obligación de generar obras o productos finales, pero sí hacer énfasis en el diálogo e intercambio de los procesos que ambos investigadores llevan a cabo para la realización de su trabajo. También una parte fundamental de este proyecto fue la sociabilización de las experiencias, las cuales fueron desarrolladas en Departamentos de la Universidad de Concepción, en donde los investigadores de las ciencias desarrollan labores docentes. Esto significó desplazar al arte a otros contextos, buscando poder ampliar el público que habitualmente participa de charlas y conversatorios.

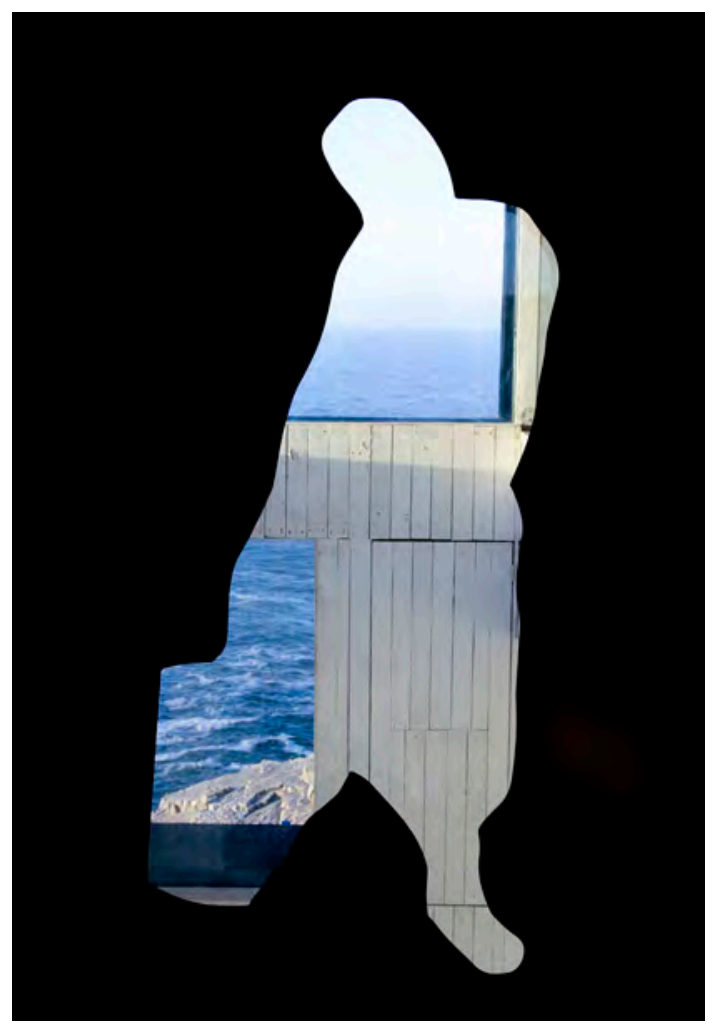




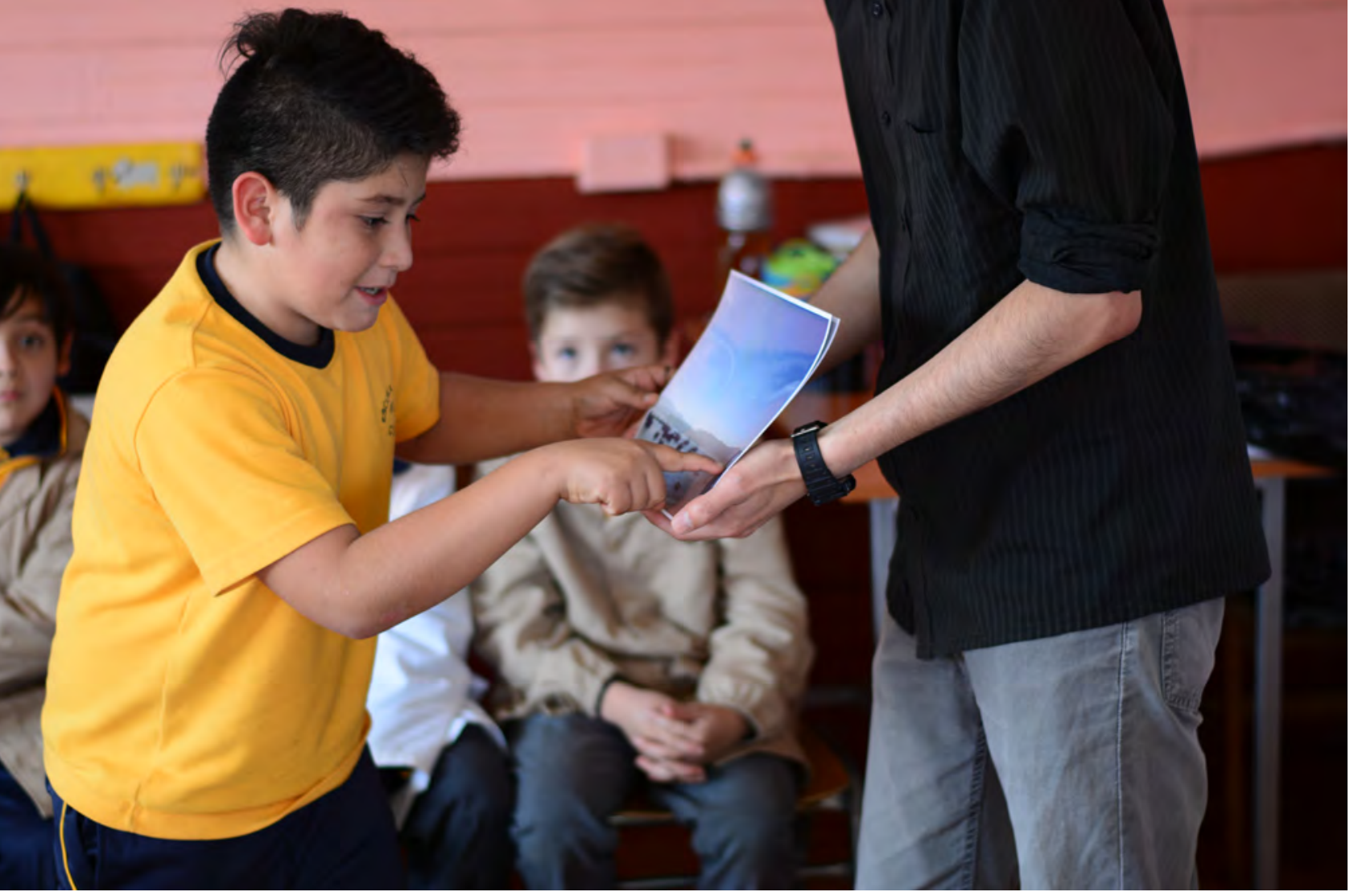

Daniel Cartes/Ciclo de residencias Territorio compartido 2016-2017, Taller Escuela Vegas de Coliumo, Marzo 2017, registro Oscar Concha.

Las dos publicaciones editadas hasta hoy a partir de las residencias realizadas han contemplado el desarrollo de textos para ampliar y generar lecturas tanto de las residencias, como figuras contemporáneas de vinculación territorial, no ajenas a cuestionamientos si consideramos que un artista instalado en un territorio siempre será foráneo. También han permitido abordar el trabajo de los artistas a partir de entrevistas interiorizándose con su procesos en el intento de conectarse con un lugar. Han participado en la elaboración de textos el historiador de arte Ignacio Szmulewicz y el teórico del arte David Romero.

Asumir el emplazamiento en un territorio es lo que hemos buscado a partir de la dirección del proyecto de residencias en Casapoli durante estos siete años. Trabajar desde este lugar situado frente a un horizonte que se enfrenta hacia el mar abierto, que nos mantiene simbólicamente conectados con el mundo pero al mismo tiempo nos involucra con una realidad muy particular. 


\section{Notas}

1 89, 91 Ochenta y nueve, noventa y uno, el proyecto Casa Poli, Mauricio Pezo, Sofía von Ellrichhausen, Eduardo Meissner, Rosmarie Prim, Ediciones CASAPOLI, Concepción, 2005.

2 Nelson Gutiérrez, profesor de música y jefe UTP de la Escuela Vegas de Coliumo G-49, Tomé.

3 A partir de esta experiencia se publica el Residente I, en conjunto con Mesa8.

4 Posterior a la residencia de Leonardo Herrera, Mesa8 publica El Residente II, que reúne parte del material gráfico y visual reunido en el Encuentro de la memoria viva, realizado en el Club de Leones de Tomé.

5 Los residentes invitados fueron Juan Castillo, Rainer Krause, Adolfo Torres, Simon Wunderlich, Colectivo Se Vende. Los artistas jóvenes seleccionados fueron Rosa Valdivia, Miguel Godoy, Alejandro Valencia, Felipe Duart.

6 Territorio compartido tuvo la participación de duplas constituidas por Francisco Navarrete Sitja y Osvaldo Ulloa, Eduardo Cruces y Andrés Tassara, Alejandro Quiroga y Javier Ramírez, Gonzalo Cueto y Álvaro Espinoza, Natascha de Cortillas y Noelia Carrasco. 